\title{
Treatment of severe deep overbite and periodontitis with residual one-third apical bone in anterior zone
}

\author{
Jiawen Zheng ${ }^{1}$ and Qing Zhao ${ }^{1}$ \\ ${ }^{1}$ Sichuan University West China College of Stomatology
}

March 2, 2022

\begin{abstract}
Although the extraordinary complex malocclusions and vulnerable periodontal status pose great challenges to orthodontists, orthodontic treatment combined with periodontal therapy is essential for patients with severe periodontitis and pathologic tooth migration. This case report describes the multidisciplinary orthodontic treatment of an adult with severe periodontitis.
\end{abstract}

\section{Introduction}

Periodontitis is a chronic inflammatory disease characterized by the progressive destruction of toothsupporting tissues. According to the global periodontal disease epidemiology, $12.5 \%$ of the population develop severe periodontitis ${ }^{1}$. Pathologic tooth migration (PTM), elongation, and spacing are common complications of adult periodontitis (AP), compromising the esthetics and function of patients ${ }^{2}$. Moreover, occlusal trauma caused by tooth migration aggravates periodontitis ${ }^{3}$. A healthy condition for AP often requires the intrusion of elongated teeth and elimination of occlusal trauma for its maintenance ${ }^{4}$. Intrusion of teeth leads to a certain gain in connective tissue attachment in the presence of adequate plaque control ${ }^{5}$.

The contraindications of orthodontic treatment for patients with periodontal disease have been widely debated $^{5,6}$. However, it has not been demonstrated clearly. Several studies have revealed that orthodontic forces per se are unlikely to convert gingivitis into destructive periodontitis ${ }^{7,8}$. The appropriate use of orthodontic force can illicit the periodontal tissue reaction desired. In contrast, orthodontic forces applied incorrectly can have a destructive effect on those tissues. Patients with periodontal disease have risk of further bone loss and tooth loss. Moreover, they often develop complex malocclusions due to the severe periodontitis. Thus, orthodontists should take special considerations when formulating the treatment plan.

For patients with severe periodontal disease, the magnitude of forces by conventional bracket and wire systems are unpredictable, which may increase the mobility of teeth. Furthermore, cutting off the brackets creates instant forces interrupting the stable status of periodontal attachment. Clear aligners overcome the shortcomings of brackets for these patients ${ }^{9}$. Precisely designed and well-calculated force contributes to maintaining healthy tissues. The aligners also relieve the occlusal trauma and distribute the forces evenly on a single loose tooth. It is less detrimental to periodontal health than fixed appliances since the aligners can be removed and allow for unimpeded oral hygiene ${ }^{10}$, which is another important advantage.

\section{Diagnosis and etiology}

The patient was a 38-year-old man with the complaint of misaligned teeth and extrusion of the maxillary incisors. He developed long-term periodontitis. Moreover, he had no systemic or congenital problems possibly affecting the treatment.

At the initial examination, a significant recession of the attached gingiva was noted on both the maxillary and mandibular anterior teeth. Deep pockets were detected almost throughout the arch using a periodontal 
probe, which demonstrated the deepest pockets around the upper and lower incisors. Both the maxillary and mandibular incisors had class III mobility. The radiograph demonstrated that the bone loss extended to the root apex of the mandibular incisors, with a poor prognosis. Vertical boss defects were noted throughout the dentition. The patient desired periodontal sequential treatment to control the bacterial plaque. When his sulcus bleeding index (SBI) and probing depth (PD) were significantly reduced to an acceptable level (Table 1), he again sought an orthodontic treatment.

On clinical examination, the patient demonstrated an excessive deep overbite with the mandibular incisors extruding to the lingual gingiva of the maxillary incisors. The bilateral molar relationship was Angle's Class I. The maxillary left lateral incisor was missing, leaving a space of approximately $5 \mathrm{~mm}$, while the mandibular dentition demonstrated moderate crowding. A 2-mm midline deviation between the face and the maxillary dentition was determined (Figure 1).

Cephalometric parameters used in this report are listed in Table 2. It confirmed that the patient had a skeletal Class II relationship (ANB: $-0.7^{\circ}$ ) with lingually inclined maxillary anterior teeth (IMPA: $74.9^{\circ}$ ). Moreover, the mandibular plane angle was in the normal range. The cephalometric analysis also demonstrated the upper lip was $4.9 \mathrm{~mm}$ behind, and the lower lip was $0.3 \mathrm{~mm}$ in front of the esthetic line (Table 2).

Panoramic radiograph showed a severely reduced alveolar bone eight, particularly in the anterior teeth area (Figure 2). The cone-beam computed tomography (CBCT) demonstrated the roots of the maxillary incisors closed to the labial alveolar bone. The bone level of the mandibular incisors was extremely reduced to the root apex (Figure 2), indicating the poor prognosis of the mandibular left incisor.

The model analysis demonstrated that there was space in the maxillary dentition, with moderate crowding in the lower dentition. The Bolton index was generally coordinated in teeth size, considering the prosthodontic therapy of the missing maxillary left lateral incisor (anterior ratio: $77.4 \%$, overall ratio: $92.51 \%$, Figure 3 ).

According to the above examination and analysis, the patient was diagnosed with Angle Class I, skeletal Class II, dentition defect, and chronic periodontitis.

\section{Treatment objectives}

The treatment objectives are as follows: 1 . Relieve the occlusal trauma and provide beneficial conditions for periodontal reconstruction; 2. Resolve the teeth with pathologic migration; 3 . Correct the severe deep bite and midline deviation; 4. Form a stable occlusal relationship; and 5. Improve the facial appearance.

\section{Treatment alternatives}

The treatment alternatives for a patient with such severe periodontal defects included orthodontic treatment, prosthodontic treatment after extraction, and only monitoring without treatment. Prosthodontic treatment had some limitations in improving the posterior occlusal relationship; even resolving the deep bite and anterior occlusal trauma appeared achievable. Extracting the natural teeth for restorative therapy resumed being unpredictable since the extremely reduced alveolar bone height and loose adjacent teeth cannot support the restoration. Occlusal trauma and periodontitis aggravated each other; therefore, no treatment, just monitoring, was negative with poor prognosis considering his age. Orthodontic treatment assisted by regular periodontal therapy was considered a better alternative to reestablish a stable occlusion and restore the esthetics and function. Finally, the patient adopted invisible orthodontic treatment and regular periodontal therapy. Clear aligners provided light and well-controlled orthodontic forces, preventing the damage from cutting off the brackets.

\section{Treatment progress}

Scaling and root planing were performed by the periodontist. Before the 3D design was processed, the following criteria had to be satisfied: well-controlled infection, percentage of bleeding after probing less than $30 \%$, and no pocket deeper than $5 \mathrm{~mm}$. The patient maintained good oral hygiene during the whole course of the orthodontic treatment. The initial staging aimed to correct the lingual inclination of the maxillary incisors and relieve the anterior occlusal trauma. When the patient wore to set 16 , the aligner in the maxillary 
incisors did not fit adequately for the existence of power ridges (Figure 4). However, the degree of derailing was within the acceptable range. When the roots of the maxillary incisors moved from the labial cortical bone to the middle of the cancellous bone, intruding was initiated. The light force was conducted, and the moving velocity was reduced to half of the normal value, which decreased the possibility and risk of looseness and teeth loss. When the treatment was processed to set 27 , the maxillary right lateral incisor aligned better from the lingual side to the dental arch (Figure 5). Moreover, the tip and torque of the maxillary central incisors were corrected significantly. Set 36 demonstrated a huge improvement of overbite after intruding the anterior teeth (Figure 6), which was more reassuring when the gingiva level and attachment loss did not get worse. After the whole aligners were worn, another set of supplementary aligners were designed to increase the intruding amount, align the mild rotated maxillary right lateral incisor and reduce the black triangles by interproximal enamel stripping (IPR).

\section{Treatment results}

At the end of the treatment, the severe deep bite was corrected using 58 sets of clear aligners, and the posterior occlusal relationship was significantly improved (Figure 7, 8, 9). The tipping and elongated incisors were aligned by lighter force and reduced stepping velocity (Figure 10). The alveolar bone height of incisors was maintained. The teeth mobility was decreased under the combined effect of regular periodontic therapy, appropriate orthodontic treatment, and oral hygiene care.

\section{Discussion}

The morbidity of periodontitis in adult patients has increased significantly ${ }^{1,2}$. The severe periodontal disease commonly results in dental disfiguration and devastates the patient's self-esteem. The prognosis of moving loose teeth is highly uncertain. The risk of tooth mobility and tooth loss is extremely high when the remaining alveolar bone is less than half the length of $\operatorname{roots}^{3,4,6}$. However, if the orthodontists do not relieve the occlusal trauma and transform the unbalanced and unhealthy pattern of mastication stress distribution, the periodontal attachment of teeth involved will absorb further and more rapidly. Atrophic alveolar bone and receding gums after tooth loss without treatment cannot support the subsequent restoration. Some research demonstrated that the combination of inflammation with occlusal trauma or tooth movement would likely produce more rapid destruction of the periodontal apparatus ${ }^{7,9}$. Therefore, regular scanning and root planing must be performed to control the periodontitis during the complete course of orthodontic treatment. Otherwise, following the intrusion, the biofilm and infection extend to the deeper area and result in a periodontal breakdown ${ }^{8}$. In this case, the alveolar bone in mandibular anterior teeth with Class III mobility only remained one-third of the root length. After moving by well-calibrated and low-magnitude orthodontic forces, the gingiva reconstructed well, and the alveolar bone was retained as before ${ }^{9,10}$. Furthermore, the mobility of involved teeth lowered to a stable level. It illustrated that severe periodontal disease did not present as the contraindication of orthodontic treatment. As long as the gingival health is achieved and maintained with periodontal therapy, intrusion of teeth conversely leads to a certain gain in connective tissue attachment in the presence of adequate plaque control ${ }^{5}$.

With light and well-controlled forces, clear aligners own definite advantages in comparison with fixed orthodontic treatment ${ }^{9}$. However, there are a few special considerations. Receding gums and long clinical crowns make the materials fill into the undercuts, which bring out the difficulty to put on or take down the aligners as well as extraction force on the loose teeth. Therefore, the edge of the aligner should be designed close to the occlusal side, which is more than $2 \mathrm{~mm}$ above the cementoenamel junction. The size of attachments should be as small as possible to reduce the retention force ${ }^{10}$. Additionally, the stepping velocity of each aligner must be lowered to avoid possible advanced periodontal involvement. The roots of patients with Angle's Class II, division 2 generally lie near the labial alveolar bone. If the intrusion of anterior teeth is designed directly at the beginning, the roots will be driven to cross the cortex, resulting in gingival recession, fenestrations, and dehiscences. It is critical to recover the inclination of anterior teeth and place the roots in the middle of cancellous bone before intruding with efficiency (Figure 11). On clinical examination, the area with deep undercuts should be approximately trimmed (Figure 12). Close attention should be paid to the occlusal trauma. In addition, it is helpful to grind the sharp dental cusps and heavy marginal ridges. 


\section{Conclusions}

Patients with PTM and occlusal trauma were treated by regular periodontal therapy and orthodontic treatment with satisfactory results. Clear aligners with well-calibrated and low-magnitude orthodontic forces corrected the severe deep bite successfully without further bone resorption, as long as the gingiva health and periodontal stabilization were maintained.

\section{Acknowledgements}

This work was supported by the National Science Foundation of China (No. 81870745 and no.81371113).

\section{Conflict of Interest}

None

\section{Authorship contribution}

Jiawen Zheng involved in case selection, details collection and draft preparation. Qing Zhao served as a senior reviewer and involved in editing.

\section{Ethical Approval}

Not applicable.

\section{Consent}

Written informed consent was obtained from the patient before the submission of the report. The signed Institutional Consent Form is on file.

\section{Figure Legends}

Fig 1. Pretreatment facial and intraoral photographs

Fig 2. Pretreatment panoramic radiograph, lateral cephalogram and cone-beam computed tomography.

Fig 3. Pretreatment study models.

Fig 4. Intraoral photographs of set 16.

Fig 5. Intraoral photographs of set 27.

Fig 6. Intraoral photographs of set 36 .

Fig 7. Post-treatment facial and intraoral photographs.

Fig 8. Post-treatment panoramic radiograph, lateral cephalogram and cone-beam computed tomography.

Fig 9. Post-treatment study models.

Fig 10. Superimposed pretreatment (black line) and post-treatment (red line) cephalometric tracings. Superimposed on the sella-nasion plane at sella.

Fig 11. Illustration of the mechanisms during the process of intruding the anterior teeth. The roots of the maxillary incisors lied near the labial alveolar bone, the intruding force through the aligners drove the roots cross the cortical bone (A). The intrusion result was predictable with approximately root lingual torque after the roots were moved to the middle of cancellous bone (B). Although the root of the mandibular incisor was in the cancellous bone, the force which was not along the tooth axis would drive the root out of the alveolar bone, resulting in gingival recession, fenestrations, and dehiscences $(\mathrm{C})$. If the intruding force cooperated with proper root lingual torque was along the axis, the tooth was probably intruded instead of lingual inclination (D). 
Fig 12. The edge of the aligner should be designed close to the occlusal side, which is more than $2 \mathrm{~mm}$ above the cementoenamel junction. On clinical examination, the area with deep undercuts should be approximately trimmed.

\section{References}

1. Nunn ME. Understanding the etiology of periodontitis: an overview of periodontal risk factors. [J]. Periodontology, 2010, 32(1).

2. Kassebaum NJ, E Bernabé, Dahiya M , et al. Global burden of severe periodontitis in 1990-2010: a systematic review and meta-regression. [J]. Journal of Dental Research, 2014, 93(11):1045-1053.

3. Towfighi PP, Brunsvold MA, Storey AT, et al. Pathologic migration of anterior teeth in patients with moderate to severe periodontitis. [J]. Journal of Periodontology, 1997, 68(10):967-72.

4. Reitan K. Effects of force magnitude and direction of tooth movement on different alveolar bone types[J]. Angle Orthodontist, 1964, 34(4):244-255.

5. Melsen B, Agerbaek N, Eriksen J, et al. New attachment through periodontal treatment and orthodontic intrusion[J]. American Journal of Orthodontics \& Dentofacial Orthopedics, 1988, 94(2):104-116.

6. Eliasson Lars-Åke, Anders H , K Jüri, et al. The effects of orthodontic treatment on periodontal tissues in patients with reduced periodontal support[J]. European Journal of Orthodontics, 1982(1):1-9.

7. Chasens AI. Periodontal disease, pathologic tooth migration and adult orthodontics[J]. The New York journal of dentistry, 1979, 49(2):40-43.

8. Sim, Hye-Young, Lee, et al. Association between orthodontic treatment and periodontal diseases: Results from a national survey[J]. Angle Orthodontist, 2017, 87(5):651-657.

9. Levrini L, Mangano A, Montanari P, et al. Periodontal health status in patients treated with the Invisalign_( system and fixed orthodontic appliances: A 3 months clinical and microbiological evaluation[J]. European journal of dentistry, 2015, 9(3):404-410.

10. Miethke RR, Vogt S. A Comparison of the Periodontal Health of Patients during Treatment with the Invisalign System and with Fixed Lingual Appliances[J]. Journal of Orofacial Orthopedics, 2005, 66(3):219-22

\section{Hosted file}

Title Page.docx available at https://authorea.com/users/463235/articles/558379-treatment-ofsevere-deep-overbite-and-periodontitis-with-residual-one-third-apical-bone-in-anteriorzone 

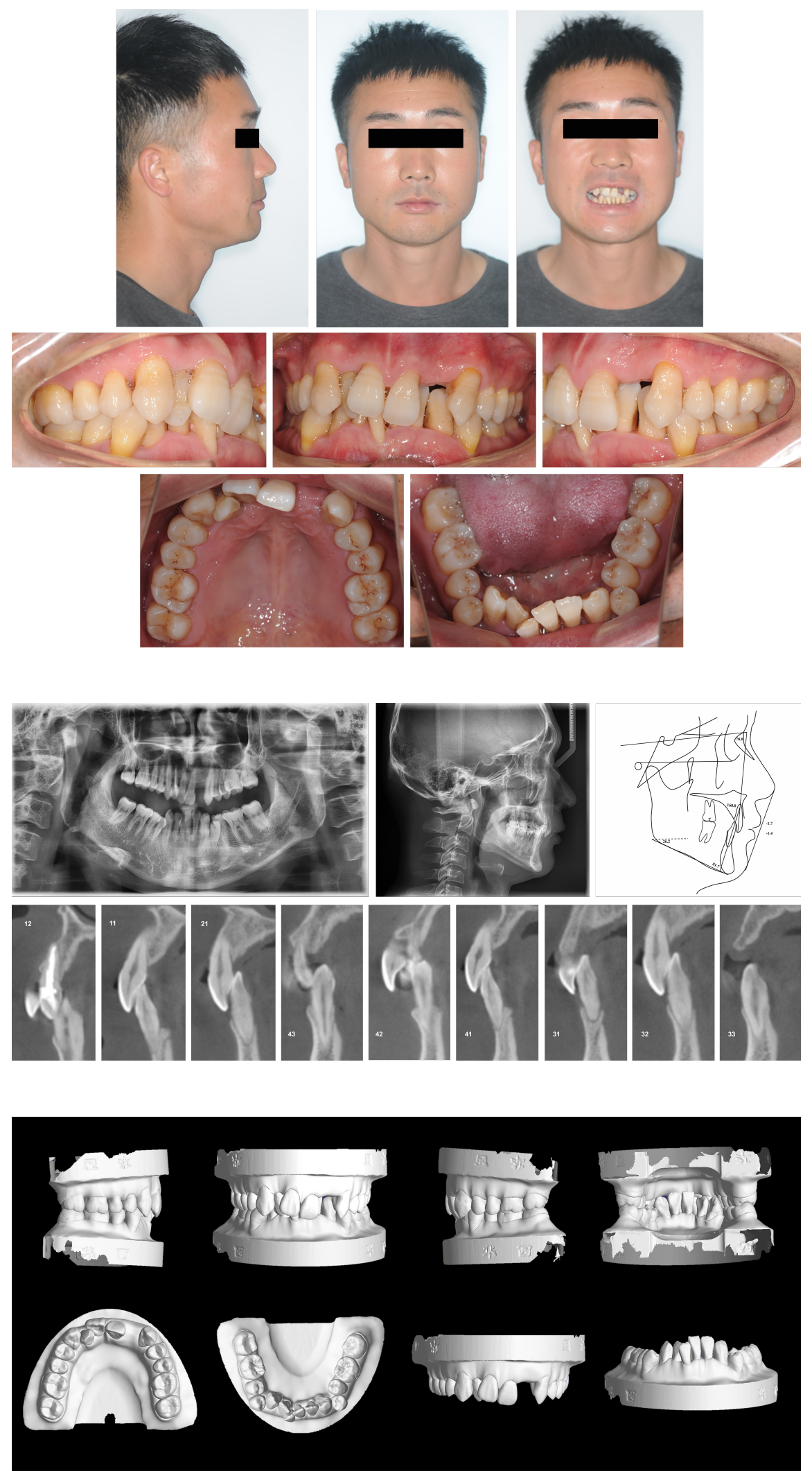

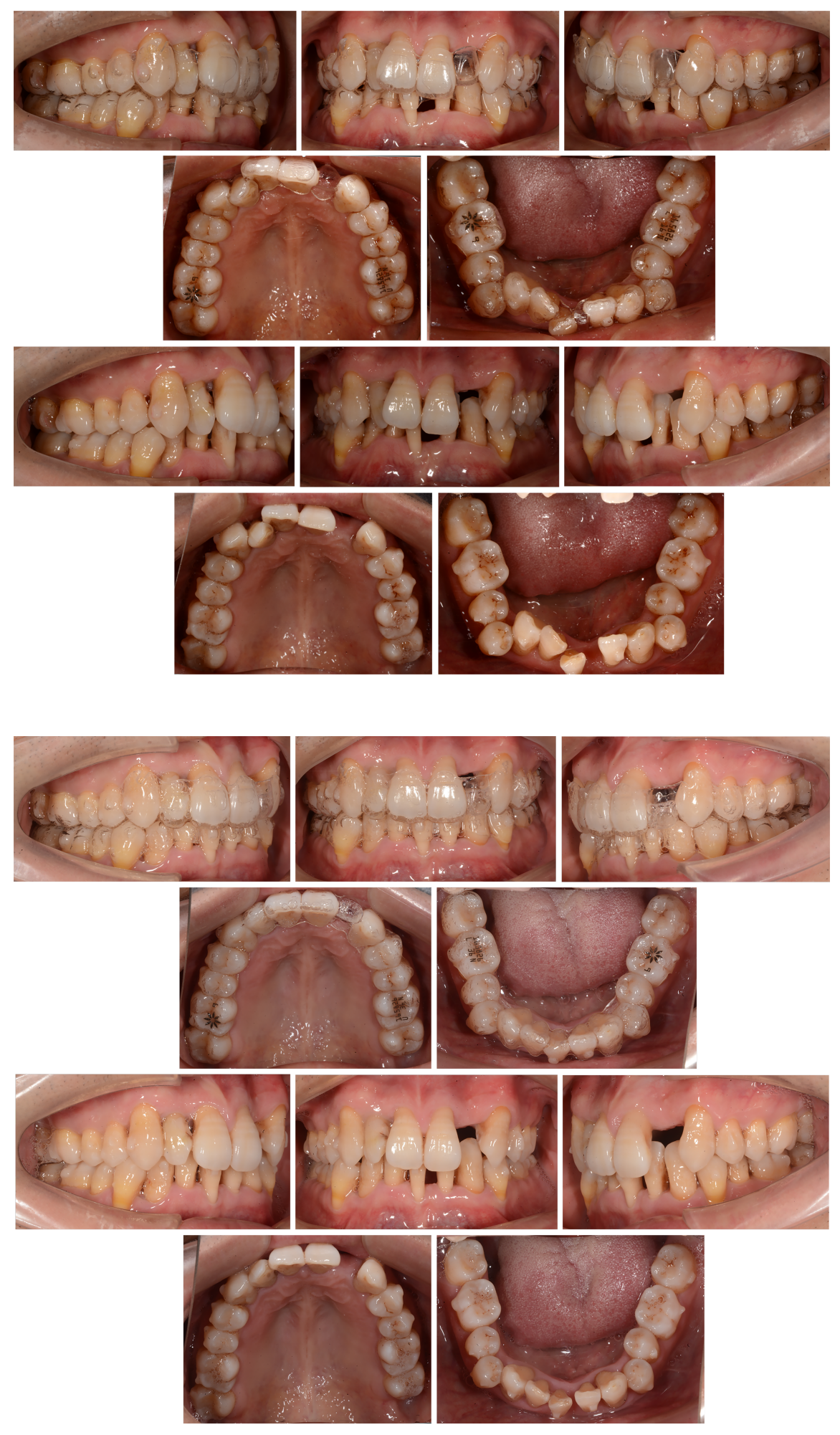

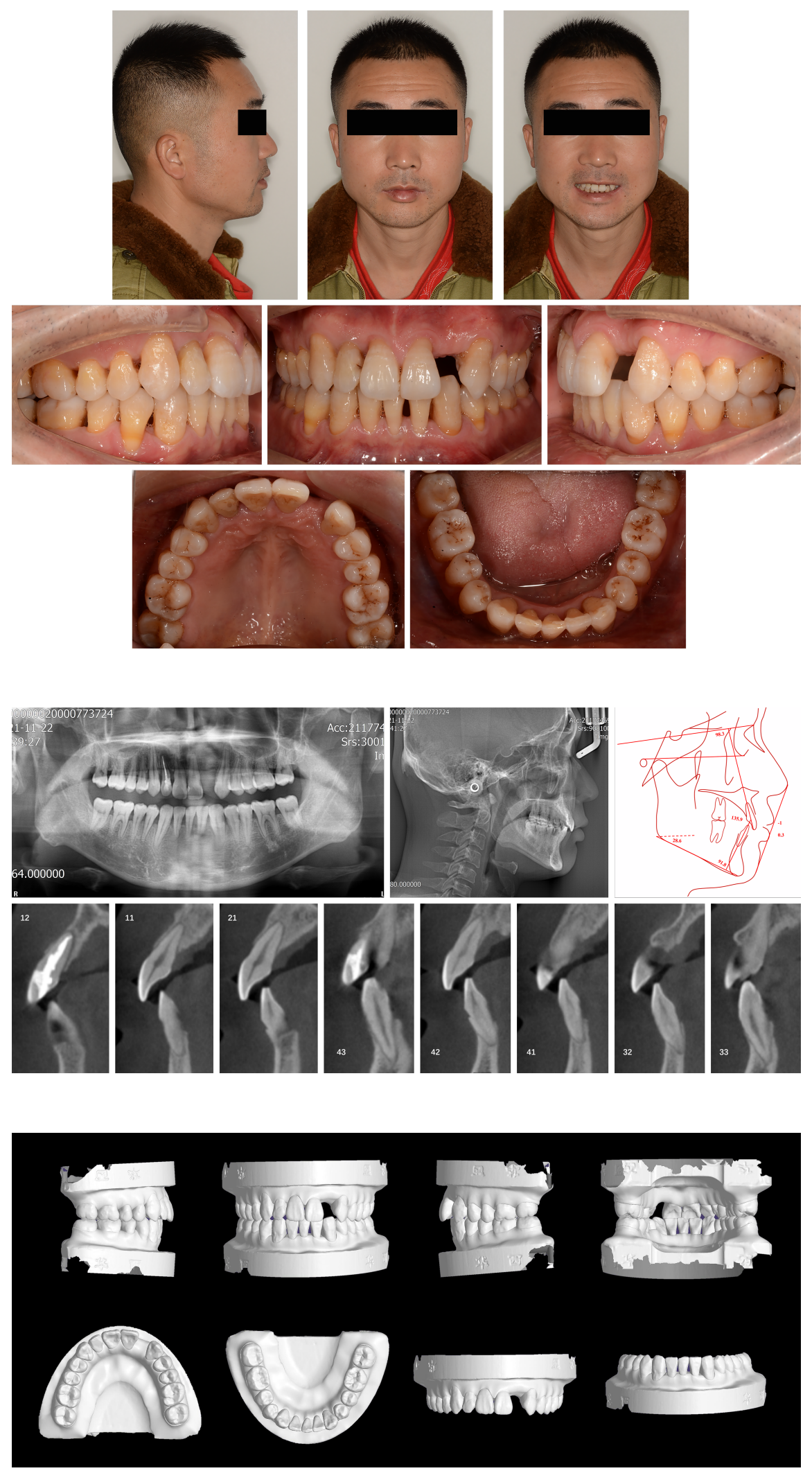

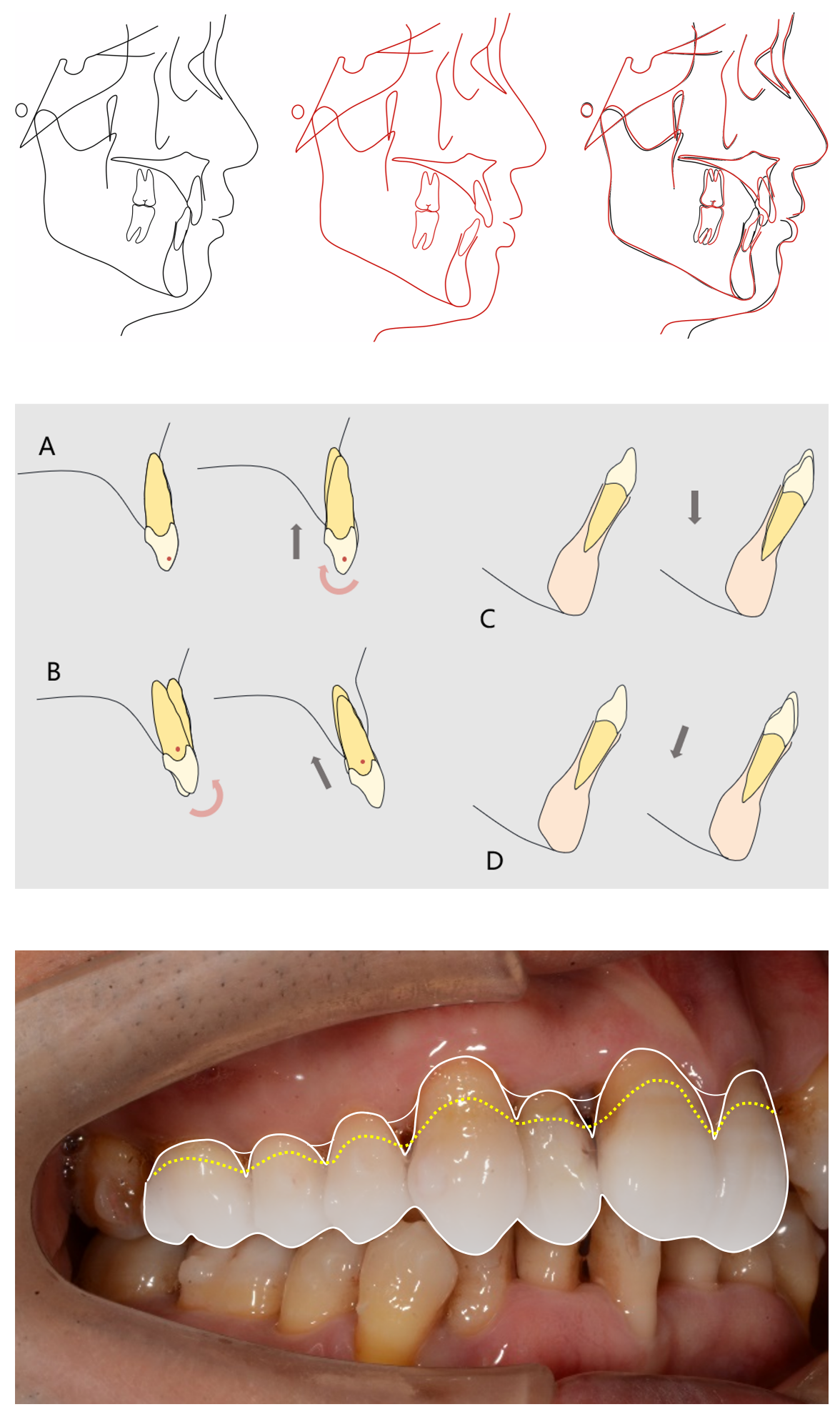